\title{
EL TRUEQUE INDIRECTO COMO MEDIDA DE RESILIENCIA EN ESCENARIOS POST-DESASTRE
}

\author{
Santiago Vélez Toro ${ }^{1, *}$ y María Luisa Eschenhagen ${ }^{1}$
}

\section{RESUMEN}

El presente artículo quiere plantear la utilización del trueque indirecto como un instrumento de intercambio de bienes y servicios, que puede ser integrado a la gestión del riesgo de desastres por ser una medida de resiliencia. El trabajo está construido en tres partes: la primera de ellas muestra los impactos sociales y económicos generados por los desastres a partir de la década de 1990 y su relación con el desarrollo, dando prioridad en la gestión del riesgo a conceptos como resiliencia y gobernanza. La segunda parte presenta el trueque de un modo general, abordando la diferencia entre los trueques directos e indirectos, exponiendo tres ejemplos de formas de intercambio alternativo. Finalmente, la tercera parte presenta el potencial que tienen los trueques indirectos para fomentar acciones de resiliencia y gobernanza, y para generar dinámicas de intercambio de bienes y servicios durante la recuperación posterior a un desastre.

\section{PALABRAS CLAVE}

Trueque, Gestión del riesgo de desastre, Resiliencia, Gobernanza

\section{INDIRECT BARTERING FOR RESILIENCE IN POST-DISASTER SCENARIOS}

\section{ABSTRACT}

The paper approaches the use of indirect barter as an exchange instrument of goods and services, and discusses this instrument as a resilience measure that could be integrated in disaster risk management. The text is structured in three sections: the first shows the social and economic impact caused by disasters since the 1990s and their relationships with development, while focusing on disaster risk management, resilience, and governance. The second section introduces general ideas about barter, addressing the differences between direct and indirect exchange through the elaboration of three examples of alternative trading. Lastly, the third part discusses the potential of indirect barter for fostering resilience and governance actions and, in addition, for generating direct and indirect exchange dynamics during a disaster recovery.

\section{KEYWORDS}

Barter, Disaster risk management, Resilience, Governance
1. Grupo Territorio, Escuela de Ciencias Sociales, Universidad Pontificia Bolivariana, Medellín, Colombia.

*Autor de correspondencia: santiago.velez.t@gmail.com

\section{RECIBIDO}

25 de febrero de 2019

\section{ACEPTADO}

11 de abril de 2019

\section{PUBLICADO}

1 de julio de 2019

\section{Formato cita}

Recomendada (APA): Vélez Toro, S. \&

Eschenhagen, M.L. (2019). El trueque indirecto como medida de resiliencia en escenarios post-desastre. Revista de Estudios Latinoamericanos sobre Reducción del Riesgo de Desastres REDER, 3(2), 17-27.

\section{(ब) $(\Theta$}

Todos los artículos publicados en REDER siguen una política de Acceso Abierto y se respaldan en una Licencia CreativeCommons Atribución-NoComercial 4.0 Internacional.

Revista de Estudios Latinoamericanos sobre Reducción del Riesgo de Desastres (REDER)

Diseño: Lupe Bezzina Tipografía: Hospital 


\section{INTRODUCCIÓN}

El presente artículo reflexiona sobre el potencial del trueque indirecto en escenarios post desastre, y surge como resultado de las reflexiones cruzadas generadas a partir de la elaboración de dos trabajos investigativos: el primero, sobre el trueque desde un enfoque de alternativas al desarrollo titulado Intercambios ¿El trueque cómo opción frente a las racionalidades del mercado? (Vélez, 2017), y el segundo, un ejercicio de investigación alrededor de los imaginarios y racionalidades que son predominantes en el Marco de Sendai (ONU, 2015), titulado Revisión del Marco de Sendai, una reflexión crítica sobre el desarrollo y la gestión del riesgo de desastres (Vélez \& Castila, 2018). Teniendo en cuenta lo anterior, el actual trabajo es elaborado a partir de un enfoque de alternativas al desarrollo, en relación directa con elementos interpretativos del pensamiento ambiental, entre estos: la crítica al desarrollo cómo modelo de crecimiento económico a través del fomento de actividades productivas y la pobreza subyacente, la necesidad de una relación armónica y de conocimiento entre el ser humano y la naturaleza, y la crítica a la concepción de la naturaleza como recurso a disposición de las necesidades humanas cualesquiera que estas sean. Estos elementos son parte de la base conceptual y teórica del presente trabajo, que permiten además considerar al desarrollo como un modelo generador de condiciones de riesgo.

En este mismo sentido, el trueque propuesto proviene conceptual y metodológicamente de los trueques indirectos que emergieron en Canadá y Estados Unidos entre 1982 y 1983 (LETS o Sistemas de Intercambio Local y Bancos de Tiempo), como respuesta a las externalidades negativas del mercado en cuanto a estabilidad económica y laboral. De esta manera, el trueque al que haremos referencia es una práctica que puede ser asumida desde el enfoque de alternativas al desarrollo, ya que promueve relacionamientos asociados a la confianza, la reciprocidad y la solidaridad, que altera las concepciones de valor de cambio y de uso al interior de los intercambios, desplazando al dinero al permitir también transacciones de todo tipo, y que al mismo tiempo, puede resultar en un elemento estructurante de la cotidianidad (como es el dinero), y también, de mercados emergentes y alternativos.

No obstante, es también importante reconocer que la gestión del riesgo de desastres es una práctica enmarcada en las racionalidades del paradigma de desarrollo, razón por la cual, este trabajo aborda las posibilidades que el trueque indirecto, entendido cómo un mecanismo alternativo de intercambio de bienes y servicios en el qué el dinero adquiere un rol secundario, tiene en términos de la gestión intersectorial consagrada en el Marco de Sendai (2015), para ser una medida de resiliencia y de gobernanza frente al riesgo de desastres. La característica. En el mismo sentido, los elementos descritos alrededor de la gestión del riesgo de desastres están influenciados por el contenido discursivo del Marco de Sendai (2015), así como por la corriente latinoamericana sobre el tema, que tiene entre sus principales referentes a autores como Alan Lavell, Cardona, Pérez y otros.

Debido a que los desastres tienen el potencial de modificar sustancialmente los medios de vida de las comunidades que impactan, promover la existencia de un sistema de intercambio alternativo indirecto es un proceso que se construye en dos momentos: el primero, cuando las comunidades conviven con el riesgo, pero deciden adoptar para sí una estrategia de preparación. Y el segundo momento, el cual es después del desastre, cuando el trueque se convierte en una verdadera alternativa de intercambio para afrontar la inestabilidad de un sistema social como consecuencia de este.

El hilo argumentativo del documento busca demostrar cómo a la luz de los impactos y la capacidad que tiene cada sociedad para recuperarse de un desastre, el factor de la pobreza es condicionante para que éstos sean más dañinos y duraderos en el tiempo, o no. Frente a esta realidad, la de una gestión del riesgo de desastres menos eficiente como resultado de un desastre, el presente artículo quiere reflexionar sobre el potencial de los trueques indirectos como una estrategia de resiliencia, la cual tiene un antes y un después en relación al desastre. Los trueques indirectos no sólo favorecen la movilización de bienes y servicios que dan un rol secundario al uso del dinero como valor de cambio, sino que al mismo tiempo generan riqueza a partir de compromisos y deudas de quienes participan en las redes de trueque. Es así, como se crean lazos de solidaridad, reciprocidad y confianza que contribuyen a establecer un tejido social resiliente. 


\section{DESASTRES Y DESARROLLO}

El desarrollo puede entenderse como un modelo generador de condiciones de riesgo (Peralta \& Velásquez, 2017, p. 68) y la probabilidad de ocurrencia de desastres aumenta a medida que el ser humano se apropia de territorios, anteriormente naturales. Cuando los territorios se modifican para dar paso a la implementación de actividades económicas, como por ejemplo la ganadería y los monocultivos de carácter extensivo, la minería de aluvión no regulada, pero también procesos de crecimiento urbano, entre otros, todas estas prácticas ocurren sobre un espacio geográfico determinado, alterando los ciclos y procesos ecosistémicos del lugar al que pertenecen. Es así como comienza a gestarse la exposición, vulnerabilidad y finalmente el riesgo frente a las amenazas presentes en ese espacio determinado. En otras palabras, las dinámicas de desarrollo pueden entenderse como el origen del proceso riesgo-desastre (Narváez et al., 2009). Para contrarrestar las externalidades sociales del desarrollo, aquellas que conjugadas con los desastres pueden impactar negativamente la forma de vida de familias y comunidades, el trueque indirecto se presenta como una estrategia de resiliencia.

Un interpretación extendida y aplicada sobre la resiliencia refiere a "la capacidad de un sistema para volver a su estructura original después de una perturbación" (Rebotier et al., 2013, p.132). Esta definición de resiliencia es asumida en la gestión del riesgo de desastres y es entendida como un proceso adaptativo positivo. No obstante, esa adaptación o capacidad para 'volver a la estructura original' no es un resultado espontaneo, requiere de preparación y acción, y es por eso también que, frente al riesgo de desastres, la resiliencia tiene un antes y un después.

Los desastres al ser eventos no proyectados producen resultados socioeconómicos complejos y difíciles de asimilar para los Estados que los sufren, sobre todo para aquellos que poseen ingresos medios y bajos. A manera de ejemplo, el informe Impacto de los desastres en América Latina y el Caribe, 1990-2011, tendencias y estadísticas para 16 países presentaba una cifra correspondiente a las pérdidas mínimas estimadas sobre viviendas durante 1999 y 2011, las que sumaban alrededor de 53.000 millones de dólares: "esta cifra resulta de avaluar en US\$20.000 el costo de reposición de una unidad básica de vivienda y en un $25 \%$, US $\$ 5.000$, la reparación de cada una dañada, sobre la base de 1.116.300 viviendas destruidas y 6.031.877 dañadas" (UNISDR \& Corporación OSSO, 2013, p.3). En relación a las muertes ocasionadas a nivel mundial por diferentes desastres, el informe titulado Poverty and Death: Disaster Mortality 1996-2015 (UNISDR \& CRED, 2016), indicaba que, en este periodo de tiempo, alrededor de 1.350 .000 personas murieron como consecuencia directa de un desastre.

La Organización de Naciones Unidas (ONU), califica los desastres como extensivos e intensivos: los extensivos son aquellos que generan un máximo de 300 viviendas afectadas y hasta 25 muertes; los intensivos son aquellos que superan esos números. La mayoría de los desastres son el resultado de fenómenos extensivos, en el informe sobre el impacto de los desastres en 16 países, mencionado en el párrafo anterior, se advierte que el $99 \%$ de los desastres reportados en éstos países en un periodo de tiempo de 22 años fueron fenómenos extensivos, "vistos de manera acumulada representan la mayoría de las personas afectadas (90\%) y de las viviendas dañadas (86\%), la mitad de las vidas humanas perdidas y el $37 \%$ de las viviendas destruidas" (UNISDR \& Corporación OSSO, 2013, p.5). Así mismo, estas cifras también indican cómo los eventos intensivos, si bien son mucho menos frecuentes, se presentan para esta zona geográfica en una relación de 1/155 (UNISDR \& Corporación OSSO, 2013), y producen impactos considerables en pérdidas de vidas y de viviendas.

Tomando como referencia el ejemplo anterior, los eventos extensivos son entonces los más recurrentes en los diferentes territorios. En jurisdicciones político-administrativas pequeñas, un evento extensivo puede tener un gran impacto sobre las dinámicas locales, sin embargo, si un evento extensivo supera las capacidades institucionales de respuesta en la escala de lo local, no debería desbordar la capacidad de respuesta en el plano regional y/o departamental. Por el contrario, los eventos intensivos que producen la pérdida de más del $60 \%$ de las viviendas y generan la mitad de las muertes en una proporción de un (1) desastre de carácter intensivo por cada ciento cincuenta y cinco (155) desastres extensivos. Por lo tanto, son el tipo de desastres que potencialmente pueden convertir a una comunidad, en más pobre y vulnerable.

En el plano mundial, el informe titulado Evaluación global sobre la reducción del riesgo de desastres. Hacia el desarrollo sostenible: el futuro de la gestión del riesgo de desastres, 2015 en adelante GAR 2015 
(Global Assessment Report 2015), estimaba que las pérdidas anuales en dólares americanos a causa de los desastres podían ser del orden de los 250.000 a 300.000 millones. Este informe también se aventuraba a predecir que para el año 2050, "el 40\% de la población mundial estará viviendo en cuencas fluviales con estrés grave por déficit hídrico" (UNISDR, 2015, p.6). A manera de ejemplo y de forma indicativa para asimilar el impacto de los desastres, basta señalar que en Colombia las Pérdidas Anuales Esperadas (PAE) por múltiples amenazas, se encuentran en lo más alto de la tabla, llegando a superar los 3.300 millones de dólares de pérdidas esperadas al año a causa de desastres (GAR, 2015, p. 65). Esta situación ubica a Colombia al lado de los gigantes del planeta en cuanto a pérdidas económicas por múltiples amenazas: Estados Unidos, Rusia, China, India, Francia, Italia, Alemania, Brasil, Australia, Japón, Irán, Perú y otros.

Las PAE son mayores en aquellos países más habitados y más desarrollados, allí, el capital expuesto es mayor y en consecuencia, los fenómenos físicos potencialmente dañinos (amenazas) generan desastres con pérdidas económicas mayores. Sin embargo, un país como Colombia, que ocupa el puesto 39 entre las economías del mundo (Dinero, 04-05-2017), con un Producto Interno Bruto (PIB) del orden de 280 mil millones de dólares (Banco Mundial, Data Bank, s/f), no posee los recursos para generar procesos de recuperación y reconstrucción eficientes en los escenarios post desastre. Por el contrario, Estados Unidos, que también pertenece a la categoría de pérdidas económicas esperadas por encima de los 3.300 millones de dólares, tiene un PIB que supera los 18 billones de dólares (Banco Mundial, Data Bank, s/f) y por lo tanto se supone que tiene una mayor capacidad de reconstrucción.

Si bien el PIB total contribuye a comprender la capacidad que tiene un país para enfrentar un desastre, el PIB per cápita constituye un indicador más aproximado para valorar las capacidades que una población tiene para hacer frente al desastre. Analizado el desastre desde esta óptica, una mayor o una menor vulnerabilidad por parte de las poblaciones expuestas a múltiples amenazas, es correlacional con el ingreso per cápita de los países. Dicho de otro modo, los países en vías de desarrollo y/o pobres, son por antonomasia, más vulnerables socialmente que aquellos países donde sus habitantes tienen mejores ingresos. Un ejemplo que refuerza este argumento es el que tiene que ver con la pérdida de vidas humanas. En el periodo de referencia 1996-2015, alrededor de 1.350.000 de personas fallecieron como consecuencia directa de los desastres. De ese gran total, 1.221.490 se ubicaron en países con ingresos bajos y medios (UNISDR \& CRED, 2016). Si alrededor del $90 \%$ de las muertes por consecuencia directa de los desastres ocurren en lugares con ingresos medios y bajos, significa que el nivel de renta es determinante para la configuración del riesgo, así como de su materialización.

Como puede observarse con las cifras presentadas, pareciera que los desastres son un asunto del desarrollo, ya que aquellos países menos desarrollados con niveles de industrialización limitados e ingresos per cápita bajos, son a la luz de los impactos sociales de los desastres, los más vulnerables al interior del sistema mundial. No obstante, es necesario resaltar que todos los países conviven con riesgos de desastres, algunos riesgos potencialmente más dañinos que otros, pero no hay espacio geográfico en el que un desastre no pueda ocurrir. Mientras la naturaleza y el ser humano se encuentren, su interacción carga con la probabilidad de desencadenar un desastre, así pues, de la conjugación de los eventos físicos dañinos (Narváez et al., 2009), entendidos como (amenazas), junto con la vulnerabilidad, entendida en función de la exposición a las amenazas que tienen la vida, los medios de vida y la infraestructura el riesgo será siempre una realidad, razón por la cual, la reducción del riesgo y la preparación de las comunidades frente al desastre no solo son acciones de resiliencia, sino también acciones de gobernanza.

Debido a que la gestión del riesgo de desastres es un asunto socialmente relevante, se convierte a su vez en un asunto de interés público y, por lo tanto, del resorte del Estado. Según Parsons (2007), las políticas públicas están directamente relacionadas con "regulación o intervención gubernamental o social" (p.37), pero también, "se puede decir que las políticas se ocupan de aquellas esferas consideradas como públicas [...] la idea de políticas públicas presupone la existencia de una esfera o ámbito de la vida que no es privada o puramente individual, sino colectiva" (p.37). Tanto la gestión del riesgo de desastres, como el manejo del desastre, son asuntos colectivos, que desbordan los intereses privados o "puramente individuales" y por lo tanto es necesario abordarlos a manera de políticas públicas.

Cuando las políticas públicas integran para sí la gestión del riesgo de desastres, y esa adopción se expresa a manera de medidas estructurales (ej. normas sismo resistentes, obras civiles de 
reducción y mitigación, etc.) y no estructurales (ej. procesos educativos, atención psicosocial, entre otras) para la reducción del riesgo, es la comunidad la que se beneficia de estas medidas. De esta manera el Estado que las impulsa, coopera en lo que se podría denominar, una gobernanza del riesgo. La gobernanza, puede ser entendida como: "Una nueva manera de gobernar que es diferente del modelo de control jerárquico, un modo más cooperativo en el que los actores estatales y los no estatales participan en redes mixtas público privadas" Mayntz (1998, citado por Cerrillo, 2005, p. 12).

Frente a unos procesos de gobierno tradicionalmente verticales y construidos de arriba hacia abajo, la gobernanza se refiere también a "procesos colectivos, formales tanto como informales, que determinan, en una sociedad, cómo se toman decisiones y se elaboran normas sociales con relación a asuntos públicos" (Hufty, 2008, p.7). Cuando los asuntos públicos vinculan de una forma no jerárquica y participativa a la ciudadanía y a los tomadores de decisiones, se puede inferir de la existencia de una buena gobernanza.

Una buena gobernanza es una práctica vital para la implementación de una adecuada gestión del riesgo de desastres, como resultado del vínculo permanente que ésta forma de gobierno promueve entre ciudadanía y Estado. Frente a la reducción del riesgo, la comunidad y el Estado deben ser aliados por un mismo fin, la salvaguarda de la vida y de los medios de vida. Para lograr esto, es fundamental reducir la vulnerabilidad, directamente ligada a la pobreza; el numeral 6 del Marco de Sendai (ONU, 2015, p.10), reconoce la pobreza cómo un "factor subyacente que aumenta el riesgo de desastres". El Marco de Sendai, del año 2015, reúne los aportes de 187 países miembros de la ONU en cuanto a la reducción del riesgo de desastres, y es a su vez, un instrumento guía para implementar acciones tendientes a reducir las condiciones de riesgo, como parte integral de la nueva agenda global, aquella que hasta el año 2030 prioriza las siguientes temáticas: desarrollo sostenible, cambio climático, nueva agenda urbana, acción humanitaria y reducción del riesgo de desastres (Peralta \& Velásquez, 2017, p. 15).

El Marco de Sendai (2015), concentra su propuesta en cuatro prioridades de acción, las cuales pueden ser resumidas así: 1- Comprensión del riesgo, 2- Gobernanza del riesgo, 3- Resiliencia, 4-Reconstrucción eficiente. Entre estas, la prioridad de acción número tres, es una propuesta que en sí misma integra nociones de gobernanza y de reconstrucción eficiente, razón por la cual, todo un abanico de nuevas formas de generar resiliencia contribuye a mejorar la relación ciudadaníaEstado en todo el proceso riesgo-desastre; en otras palabras, las medidas de resiliencia actúan antes y después del desastre. Dicho esto, el trueque indirecto cómo práctica resiliente, tiene que ver con la gobernanza del riesgo en un escenario previo a un desastre; pero también, está relacionado con responder eficazmente y reconstruir mejor.

Frente al riesgo, entendido como una probabilidad de impactos negativos sobre un sistema social, tener un respaldo para favorecer los intercambios es una medida de resiliencia. No solo se está en total sintonía como el Marco de Sendai (2015) en cuanto a preparación, gobernanza y reconstrucción, sino que también contribuye a fortalecer otras racionalidades económicas entre los individuos que hacen parte de redes de trueque.

Cuando el riesgo se materializa y el desastre modifica sustancialmente las realidades sociales, económicas y de aprovisionamiento de una comunidad, el trueque indirecto tiene el potencial de dinamizar redes de intercambio local que no se centran en el dinero como medio de cambio socialmente aceptado, sino en preceptos tales como la solidaridad, la reciprocidad y la confianza (Álvarez et al., 2010). Así, el trueque a la manera en que se plantea en este documento es una práctica que contribuye a la construcción de tejido social, porque desplaza al precio como medida de valor y de acceso a un bien o un servicio, y lo sustituye por una medida de valor que reconoce el tiempo, la dificultad, la solidaridad, el conocimiento y todo un sinfín de condicionantes que hacen posibles intercambios que en el mundo del dinero serían improbables. El trueque que se quiere presentar en este documento tiene que ver con un trueque como complemento a un mercado colapsado o inestable como resultado de un desastre.

\section{TRUEQUES INDIRECTOS, EJEMPLOS PRÁCTICOS}

En términos generales existen dos tipos de trueques: el primero el trueque simple (Harvey, 2014), también llamado trueque directo. En este tipo de trueque, la "no coincidencia de intereses" (Harvey, 2014, p.41) o "la imposibilidad el trueque generalizado" (Kicillof, 2013, p.67), ha sido desde siempre y continúa siéndolo en el presente, una dificultad para que más truequeables (entendidos como 
bienes y servicios, también mercancías, elementos que pueden ser intercambiados) puedan ser movilizados. Un ejemplo para entender esta dificultad es el siguiente: "una persona (A) quiere intercambiar un producto por el producto de una persona (B), sin embargo, (B) no demuestra interés por el producto de (A). Bajo estas condiciones, el intercambio es imposible" (Vélez, 2017, p. 66). Lo que puede observarse en este ejemplo es la imposibilidad de llevar a cabo un intercambio, así las dos personas involucradas tengan el interés de que este se concrete. Adicional a esta dificultad, mientras más personas entran en la ecuación, más complejo es el intercambio, ya que, como lo anota Harvey (2014) "para que se produzca un trueque simple, otro ha de poseer algo que yo deseo y yo he de tener algo que el otro desea. Se pueden construir cadenas de trueque, pero son limitadas y engorrosas" (p.39). De la frase anterior se desprende que cuando se privilegian trueques directos, la movilidad de los truequeables -que también pueden ser entendidos a manera de mercancías- es menor, por lo tanto:

"El trueque directo limita las posibilidades de cambio a los bienes y servicios existentes y la cantidad de estos, sin contar que hay bienes y servicios más demandados que otros por los integrantes de un club o red de trueque. Cuando el trueque directo se agranda e implica un grupo de personas cargado de necesidades por ser satisfechas en el mercado, la dificultad del intercambio aumenta, y es entonces cuando este sistema económico pierde relevancia y practicidad frente al dinero"

Vélez (2017, p.66)

Entonces, ya que el trueque directo es limitado y engorroso, y no parece poseer las cualidades para movilizar intercambios que involucren a grupos de personas, se podrían explorar otro tipo de trueques, aquellos que son indirectos y que utilizan monedas alternativas. Trueques que han emergido a partir de la segunda mitad del siglo XX y que se presentan como evidencias de formas plausibles de realizar intercambios alternativos.

Admitiendo que los trueques pueden ser entendidos como todos aquellos intercambios en donde el poder de compra del dinero no juega un rol trascendental a la hora de promover y realizar intercambios de todo tipo, se presentarán a continuación tres ejemplos de prácticas que pueden ser consideradas cómo trueques indirectos.

La primera práctica involucra el uso de monedas alternativas, que son un elemento de los trueques modernos y también parte de los trueques indirectos. Una definición posible de monedas alternativas es la siguiente: son "sistemas monetarios sociales, también conocidos como alternativos, comunitarios, complementarios [...] a aquellos sistemas monetarios que se crean al margen de las monedas oficiales del país, y que tienen como objetivo fundamental la promoción de proyectos económicos y sociales de carácter local" (CAJAMAR, 2008, p.17). Siguiendo a Santana (2011, pp.264-265), existen cuatro rasgos que motivan el surgimiento de monedas alternativas, también llamadas facilitadores: a) Ayudan a remunerar el trabajo, teniendo o no un empleo; b) Facilitan los intercambios en ausencia de dinero, o cuando se tiene disponible poco dinero; c) Previene la escasez en catástrofes financieras; y d) Promueve la inclusión social a través de intercambios recíprocos.

Teniendo formas de funcionamiento propias, las monedas alternativas cumplen el rol de armonizar 'la no coincidencia de intereses' con la necesidad de movilizar los truequeables (entendidos como todo lo factible de ser intercambiado). En ese sentido, estas 'otras' monedas, desempeñan un papel similar al dinero ya que tienen poder de compra, y "se le conoce también como dinero social por ser creado, emitido y controlado por grupos sociales y dinero comunitario en parte, porque funciona en comunidades de personas que se relacionan directamente y desean establecer un medio de intercambio alternativo (Santana, 2011, p. 265). Resultado de lo expresado por Santana (2011), se puede inferir que los trueques que utilizan moneda alternativa y promueven intercambios alternativos, responden a contextos y realidades que expresan la necesidad de involucrar a los excluidos en dinámicas de intercambios (Francés et al., s/f, p. 12). Esto facilita el acceso a bienes y servicios, y el mejoramiento de condiciones de vida para aquellas personas con dificultades de acceder al dinero como medio de compra.

Otra práctica de trueque indirecto son los Bancos de Tiempo. Estos emergieron en Estados Unidos en 1983, el primero de ellos, conocido como Time Dollars, facilitaba a la población 
tradicionalmente marginada, como los ancianos, los pobres, los desempleados, entre otros, acceder a ciertas redes de comercio, utilizando como medio de cambio la hora de trabajo. En los Bancos de Tiempo la unidad de valor es una hora, ya que "las horas de un operario sin cualificación valen lo mismo que las de un directivo con alta cualificación académica y profesional" (CAJAMAR, 2008 , p.42). Con esta orientación respecto al tiempo como unidad de valor, el funcionamiento de los diferentes Bancos de Tiempo es más o menos el mismo.

Los Bancos de Tiempo se caracterizan por tener una entidad reguladora que imprime una moneda, cuyo valor en tiempo equivale al de una hora y con esta moneda se le paga a quien realiza un trabajo en horas al interior de la red. Uno de los Bancos de Tiempo más reconocido es el que funciona en Ithaca (Nueva York), diseñado y puesto en funcionamiento por Paul Glover en 1991, quien "decidió poner en marcha este dispositivo para tratar de alentar a la población a que utilizara su tiempo y dinero dentro de la comunidad y frenar así lo que él consideró como un desvió de energía hacia la gran ciudad de Nueva York" (Francés et al., s/f, p. 10). La moneda creada por Glover, el Ithaca Hours, poseía un valor de compra equivalente a 10 dólares, esté podía ser utilizado en un radio de 20 millas tomando como base el centro de la localidad. Para acceder a esta moneda alternativa era necesario intercambiar trabajo por tiempo, y debido a que una gran parte de la comunidad hacía parte del Banco de Tiempo, se diseñaban diferentes y continuas estrategias por parte del emisor, para que las personas pudieran acceder a la moneda alternativa, pudiéndolas usar como medio de cambio. Según Moers (1998), el éxito de este Banco de Tiempo fue que logró involucrar al comercio local en la dinámica de intercambio alternativo, razón por la cual la moneda Ithaca Hours adquirió legitimidad entre la población: la certeza de saber que era una moneda alternativa con un valor de cambio real generaba en la comunidad la confianza suficiente para utilizarla.

Finalmente, la tercera práctica de trueque indirecto, los LETS, traducidos al español como Sistemas de Intercambio Local, fue creada por Michael Linton en Canadá, en 1982, cuya motivación fue la de generar una respuesta económica al desempleo de la región que habitaba (Francés et al., $s / f, p$. 9). Funcionaban como una red, en la cual, los participantes valoraban en una moneda común, generalmente en una relación uno a uno con el dólar, los bienes y servicios que estaban disponibles para el trueque. Lo interesante de esta red, es que la satisfacción inmediata por el intercambio podía ser pospuesta; en otras palabras, un participante podía solicitar un bien o un servicio de alguien más, cómo por ejemplo reparar una puerta, y quedar en deuda con la red, así mismo, la red en su conjunto quedaba en deuda con quien otorgaba su bien o servicio. En este tipo de trueque se crea riqueza a partir de la deuda generada.

Para el correcto funcionamiento del sistema LETS, la existencia de cuentas con balance negativo es absolutamente necesaria pues solo así es posible la creación de riqueza, ya que se trata de un juego de suma cero en el que el total de los balances negativos es exactamente igual al de los balances positivos. Así, cuanto mayor sea el poder de endeudamiento, mayor será la capacidad de creación de riqueza por parte del grupo (Francés et al., s/f, p.10). Para controlar el exceso de endeudamiento por parte de las personas de la red, los LETS se centralizaron e hicieron pública la información sobre los balances positivos y negativos de sus miembros. De esta forma, después de un periodo de tiempo concertado, por ejemplo, de 6 meses, se realizaban acciones de compensación social para generar una dinámica de reciprocidad entre quienes tuvieran balances negativos y positivos. Así se garantizaba un cierto equilibrio a la red.

Sin embargo, los LETS, corrían el riesgo de dejar de funcionar si algún miembro de esta red dejaba de cancelar sus deudas (Schuldts, 1997, citado por Francés et al., s/f). Por la razón anterior, la importancia de promover el sistema LETS en entornos locales, donde los miembros se conocen, es lo que imprime confianza en el sistema, y favorece las acciones de trueques como actos solidarios y recíprocos.

\section{POTENCIAL DEL TRUEQUE EN ESCENARIOS POST DESASTRE}

Después de explicar estas tres formas diferentes de trueques indirectos, es relevante señalar cómo estas prácticas alternativas tienen el potencial de promover intercambios de todo tipo y, sobre todo, en todo tipo de circunstancias. Visto desde esta óptica, la puesta en marcha de trueques indirectos como medida de resiliencia, de gobernanza y de preparación para afrontar un desastre, es también una práctica con el potencial de construir tejido social en torno a tres valores esenciales: la solidaridad, la reciprocidad y la confianza (Álvarez et al., 2010).

En la fase de la preparación ante desastres, es el momento para que las comunidades apropien para sí los trueques indirectos, desde lo metodológico y desde lo práctico, de modo tal 
que puedan responder adecuadamente en el momento de la emergencia o el desastre. Para que un trueque indirecto se logre sostener en el tiempo es importante que entre quienes participan de la red existan niveles de confianza comunitaria, esto es esencial para generar riqueza y movilidad de bienes y servicios a partir de una deuda. Por lo tanto, la apropiación social del trueque indirecto por parte de las comunidades es preferiblemente un acto de preparación, una acción anterior a la materialización del riesgo

Ese periodo previo al desastre, el de la convivencia de los sistemas sociales con el riesgo, es también el momento en donde la gobernanza toma su forma, implicando procesos de preparación a través de la participación. Cómo fue mencionado en párrafos anteriores, la gobernanza hace referencia a ejercicios de toma de decisión menos jerárquicos, en los cuales hay unos procesos colectivos que desembocan en acciones que tienden a gobernar bien. Para Hufty (2008), estos procesos colectivos pueden ser formales o informales, en esa medida, el trueque que se construye de abajo hacia arriba puede ser informal, pero también formal, cuando el Estado y quienes lo administran comprenden que este sistema promueve espacios de participación y solidaridad ciudadana, convirtiendo a los individuos en sujetos políticos conscientes de su rol en los procesos de desarrollo.

Además, para que los trueques indirectos puedan emerger y sostenerse en el tiempo, es necesario que exista una forma compartida entre los miembros de la red de otorgar valor a todos aquellos bienes y servicios que puedan ser intercambiados. Lo anterior es importante tenerlo presente, ya que en la economía de mercado el intercambio normalmente se reduce a un ejercicio de vender y comprar utilizando como mediador de la transacción el dinero, a diferencia del trueque, en donde el compromiso de hacer circular las mercancías determina la posibilidad de que dos o más bienes y servicios puedan ser intercambiados para satisfacer necesidades concretas e inmediatas, así estos no sean equiparables en su precio en el mundo monetizado de la economía de mercado. Adicionalmente, el trueque según Hintze (2003), Coraggio (2011) y Gatti (2010), permite el restablecimiento de lazos de solidaridad entre vecinos, al dignificar el trabajo, a través de un reconocimiento del tiempo que se toma la producción de un bien o un servicio.

En este orden de ideas, el trueque se presenta como un mecanismo propicio para establecer intercambios cuando el sistema económico de mercado es impactado negativamente a consecuencia de un desastre. Estos pueden ocasionar, además de las pérdidas en vidas y materiales, un colapso financiero, el cual puede reducir la cantidad de dinero circulante, limitando el poder de compra de las personas: pero, además, un desastre puede romper con cadenas productivas y con ello propiciar el desabastecimiento y el desempleo. Ya sea porque el mercado se debilita a causa de un desastre, o porque un desastre afecta la capacidad de personas y grupos de personas para acceder normalmente a las dinámicas de la economía de mercado, el trueque puede contribuir a que estas poblaciones vulnerables puedan adherirse y tener espacios de intercambio de bienes y servicios, sin la utilización de dinero, o con muy poco dinero.

Como fue señalado anteriormente, más del $90 \%$ de las muertes ocasionadas por desastres ocurren en países en vía de desarrollo, esto permite deducir que la pobreza desempeña un rol importante alrededor de la implementación de medidas de gestión del riesgo de desastres. Siendo la pobreza uno de los mayores obstáculos para la respuesta eficiente en escenarios post desastre, el trueque indirecto podría ser asumido como medida de resiliencia. Esto es posible debido a que sea cual fuere el mecanismo utilizado (LETS, Bancos de Tiempo, Monedas Alternativas), el intercambio indirecto contribuye al aumento de capacidades de las comunidades, al presentar una forma alternativa de movilizar intercambios. Es decir, cuando las comunidades adoptan para sí, el conocimiento conceptual y práctico alrededor de formas alternativas de generar intercambios de bienes y servicios, estas comunidades reducen sus niveles vulnerabilidad. A manera de ejemplo negativo sirve considerar lo ocurrido en Haití del año 2010, "un terremoto de 7.0 de magnitud, golpea Haití dejando 222.570 muertos, 300.572 heridos y 2.3 millones de personas desplazadas" (IFRC, 2014, p.2). Haití es un ejemplo de la manera como un desastre que ocurre en una población previamente vulnerable al ser muy pobre, y sin redes de trueque, lo cual la hace más vulnerable cuando el evento ocurre. Esta vulnerabilidad continúa aumentando en la medida que los procesos de reconstrucción y recuperación son poco eficientes.

Mientras que el caso de Japón es un ejemplo de "reconstruir mejor" (ONU, 2015, p. 21), también por tener un nivel de pobreza menor y una estructura de solidaridad mayor, lo cual facilitó en este 
país después de un desastre las acciones de reconstrucción. Condiciones que tienden a reducir al máximo la vulnerabilidad tanto física como social de las áreas afectadas. Por el contrario, Haití es un ejemplo de la manera cómo un desastre que ocurre en una población previamente vulnerable al ser muy pobre, la hace aún más vulnerable cuando el evento ocurre, y la vulnerabilidad continúa y se potencializa, aumentando en la medida que los procesos de reconstrucción y recuperación son poco eficientes. Por lo tanto, estas asimetrías entre países industrializados y los que no lo son, reflejan diferencias significativas, en cuanto a recuperar y reconstruir eficientemente y mejor, reforzando la noción de que el desastre y la recuperación posterior es un asunto de desarrollo.

La pobreza en países en vías de desarrollo genera y reproduce de forma permanente condiciones de vulnerabilidad, acrecentando el riesgo de desastres. La pobreza a su vez se manifiesta a través de las posibilidades de acceso limitado a bienes y servicios básicos para vivir dignamente. El trueque, si bien no constituye un sistema en capacidad de sustituir la economía de mercado en toda su extensión, si tiene el potencial de promover intercambios con reglas que pueden adaptarse a diferentes coyunturas. En otras palabras, el trueque es un complemento al mercado con potencial de generar intercambios en escenarios post desastre. Al reconocer que los trueques son complementos al mercado, es importante señalar que este sistema puede movilizar transaccionalmente bienes y servicios, al margen de las formas monetaristas de la economía de mercado, al favorecer intercambios de todo tipo, sin el uso del dinero como valor de cambio. Así pues, frente a esta realidad de dominancia de la economía de mercado y la vulnerabilidad que supone tener una sola forma de intercambio económico, en comunidades pobres que conviven con el riesgo de desastres, los trueques indirectos pueden implementarse como estrategias de resiliencia para contrarrestar las vicisitudes del mercado. Esta estrategia debería ser considerada, sobre todo en aquellas zonas proclives a los desastres y en donde los estudios de riesgo han identificado comunidades que se encuentran en zonas de amenaza alta, ya sea por inundación, avenidas torrenciales, sismos, movimientos en masa u otros eventos físicos potencialmente dañinos.

\section{CONCLUSIONES}

La gestión del riesgo de desastres es, tanto una realidad, como una necesidad en un mundo en desarrollo. En este sentido, la generación de estrategias territorialmente contextualizadas que permitan preparar a la población para afrontar eventuales desastres es una medida de gobernanza y también de resiliencia. Este artículo se propuso introducir el potencial del trueque para ser un instrumento a tener en cuenta en la gestión del riesgo de desastres, al abordar un tema especialmente sensible del andamiaje actual del desarrollo, la economía de mercado y la circulación de bienes y servicios. El trueque entonces es una manera de promover intercambios cuando las condiciones del mercado resultan contrarias a su funcionamiento habitual. Ejemplos como el sistema LETS y los Bancos de Tiempo ofrecen experiencias que emergen como respuesta a mercados inestables y, sobre todo, como respuesta a mercados inaccesibles para aquellos más vulnerables. Dicho esto, este artículo propone considerar una adaptación de los trueques indirectos a las lógicas de la gestión del riesgo de desastres, esa que de forma integral y multidisciplinar busca reducir el impacto negativo de un desastre a partir de procesos de preparación.

Los desastres tienen impactos mayores en el mundo considerado 'en vías de desarrollo', en otras palabras, en el mundo pobre, no porque los daños superen en costos aquellos ocurridos en el mundo desarrollado, sino porque estos países están en condiciones de inferioridad para movilizar los recursos necesarios para procesos de reconstrucción eficientes en escenarios post desastre. Estas asimetrías entre países industrializados y los que no lo son, reflejan diferencias en cuanto a recuperar y reconstruir eficientemente y mejor, reforzando la noción de que el desastre y la recuperación posterior es un asunto de desarrollo, o de falta de este, según cómo se mire.

Cuando el acceso de las personas al mercado se encuentra limitado por condiciones de pobreza su vulnerabilidad se acrecienta. Si a esta vulnerabilidad inicial se le adiciona la exposición a una amenaza, el subsiguiente riesgo y la ocurrencia de un desastre, la situación puede de forma potencial, perturbar en el corto, mediano y largo plazo los medios de vida de los afectados. Frente a esta realidad socio económica, de interacción permanente de la sociedad con la naturaleza, el trueque indirecto es una alternativa práctica para movilizar intercambios de todo tipo en situaciones, donde la estabilidad del mercado sea alterada por escenarios tales como los desastres.

Finalmente, la promoción de redes de trueques indirectos podría propiciar el fortalecimiento del tejido social comunitario, al tiempo que favorecen la relación ciudadanía-institucionalidad, 
cuando es esta última la que moviliza estrategias de resiliencia. Además, el trueque indirecto contribuye a preparar a las personas para que, en caso de un desastre, puedan seguir generando flujos de bienes y servicios mientras la normalidad retorna.

\section{REFERENCIAS}

Álvarez, S., Bordón, M.L., González, R. \& Valeri, P. (2010). Microcréditos en la economía social. Experiencia del Banco Popular de La Buena Fe "Sol de Otoño", ciudad de Neuquén. Neuquén: FONCAP. Recuperado de: https://www.academia.edu/1902733/Microcr\%C3\%Agditos_en_ la_Econom\%C3\%ADa_Social._Experiencia_del_Banco_Popular_de_la_Buena_Fe_Sol_de_ Oto\%C3\%B1o_ciudad_de_Neuqu\%C3\%A9n?auto=download [visitado o4 de agosto de 2019]

Banco Mundial. (2018a). Datos: Colombia [Datos]. Recuperado de: https://datos.bancomundial.org/ pais/colombia. [visitado o6 de Sep. de 2018]

Banco Mundial. (2018b). Datos: Estados Unidos [Datos]. Recuperado de: https://datos.bancomundial. org/pais/estados-unidos?view=chart [visitado o6 de Sep. de 2018]

Campos, A., Holms-Nielsen, N., Díaz, C., Rubiano, D., Costa, C., Ramírez, F. \& Dickson, E. (2012). Análisis de la gestión del riesgo de desastres en Colombia. Un aporte para la construcción de políticas públicas. Bogotá: Banco Mundial Colombia \& GFDRR. Recuperado de: http://repositorio. gestiondelriesgo.gov.co/handle/20.500.11762/18426 [visitado 04 de agosto de 2019]

Cerrillo, A. (coordinador). (2005). La gobernanza hoy. 10 textos de referencia. España: INAP.

Coraggio, J. L. (2011). Economía social y solidaria. El trabajo antes que el capital. Ecuador: Abya-Yala.

Dinero. (2017). Las economías más grandes del mundo ¿cómo se distribuye el PIB a nivel global? [Web]. Recuperado de: https://www.dinero.com/economia/articulo/economias-mas-grandes-del-mundopor-tamano-del-pib/243710 [visitado o6 de Junio de 2018]

Federación Internacional de Sociedades de la Cruz Roja y de la Medialuna Roja IFRC. (2015). Terremoto de Haití. Informe de avance de 5 años. Ginebra: IFRC. Recuperado de: https://www.ifrc.org/Global/ Documents/Americas/201501/1287600-IFRC-Haiti\%205-year\%2oprogress\%2oreport-SP-LR.pdf [visitado o6 de Sep. de 2018]

Francés, F., García, J.T. \& Aris, L. (s/f). Nuevos sistemas de intercambio comunitario: un ejemplo de glocalización. Recuperado de: https://studylib.es/doc/5349529/nuevos-sistemas-de-intercambiocomunitario--un [visitado o4 de agosto de 2019]

Fernández, M. (2009). El trueque solidario. Una estrategia de supervivencia ante la crisis argentina de 200o. Revista Pueblos y Fronteras Digital, 4(7), 5-29.

Gatti, C. (2010) El fenómeno del trueque: una mirada sociológica. Revista pueblos y fronteras digitales, $5(8), 1-23$.

Harvey, D. (2007). Breve historia del neoliberalismo. Madrid:Ediciones Akal

Harvey, D. (2014). 17 Contradicciones del Capitalismo. Quito \& Madrid: Profile Books \& IAEN

Hintze, S. (2003). Trueque y economía solidaria. Buenos Aires: Prometeo Libros.

Hufty, M. (2008). Una propuesta para concretizar el concepto de gobernanza: el marco analítico de la gobernanza. La Paz: IFEA-IRD.

Kicillof, A. (2013). De Smith a Keynes. Siete lecciones de historia del pensamiento económico. Un análisis de los textos originales. Buenos Aires: Eudeba.

Moers, P. (1998). Community currencies: A co-operative option for the developing world? Appropriate Economics [Web]. Recuperado de: https://appropriate-economics.org/materials/CCS_-_A_ Cooperative_Option_for_the_Developing_World.pdf [visitado o4 de agosto de 2019]

Narváez, L. Lavell, A. \& Pérez, G. (2009). La gestión del riesgo de desastres. Un enfoque basado en procesos. Lima: Secretaría General de la Comunidad Andina.

Organización de Naciones Unidas ONU. (2015a). Marco de Sendai para la reducción del riesgo de desastres 2015-2030. Sendai: ONU.

Organización de Naciones Unidas ONU. (2015b). Informe Global sobre Reducción del Riesgo de Desastre GAR 2019. Evaluación global sobre la reducción del riesgo de desastres. Hacia el desarrollo sostenible. El futuro de la gestión del riesgo de desastres. Ginebra: ONU.

Parsons, W. (2007). Políticas Públicas. Una introducción a la teoría y la práctica del análisis de políticas públicas. Ciudad de México: FLACSO México. 
Peralta, H.\& Velásquez, A. (2017). Resiliencia. La clave del nuevo liderazgo del siglo XXI. Bogotá: Gráficas Santa María SA.

Pérez, J.C., Etxezarreta, E. \& GoridI, L. (2008) ¿De qué hablamos cuando hablamos de economía social y solidaria? Concepto y nociones afines. Economía Solidaria [Web]. Recuperado de: https://www. economiasolidaria.org/sites/default/files/reaslibrary/attachments/Economia_social_y_solidaria_ concepto_nociones.pdf [visitado 21 de junio de 2018]

Programa de las Naciones Unidas para el Desarrollo PNUD. (s/f). China: recuperación temprana: reconstruir mejor y más verde después del terremoto. PNUD [Web]. Recuperado de: http://www. undp.org/content/undp/es/home/ourwork/ourstories/china--building-back-better-and-greener. html [visitado o4 de agosto de 2019]

Rebotier, J., López Peláez J. y Pigeon P. (2013). Las paradojas de la resiliencia: miradas cruzadas entre Co- lombia y Francia. Territorios, 28, 127-145.

Santana, M.E. (2012). Recrear el dinero en una economía solidaria. Revista polis, 29. Recuperado de: http://journals.openedition.org/polis/2005 [visitado o4 de agosto de 2019]

UNISDR y Corporación OSSO. (2013). Impacto de los desastres en América Latina y el Caribe, 19902011. Tendencias y estadísticas para 16 países. Ciudad de Panamá: UNSDR \& Corporación OSSO. Recuperado de: http://eird.org/americas/noticias/Impacto_de_los_desastres_en_las_Americas.pdf [visitado o4 de agosto de 2019]

UNISDR y Centre for Research on the Epidemiology of Disasters CRED. (2016). Poverty and Death: Disaster Mortality 1996-2015. Geneva: UNSIDR: CRED. Recuperado de: https://www.unisdr.org/ files/50589_creddisastermortalityallfinalpdf.pdf [visitado o4 de agosto de 2019]

Vélez, S. (2017). Intercambios ¿El trueque como opción frente a las racionalidades de la economía de mercado? Tesis de Maestría. Medellín: Universidad Pontificia Bolivariana.

Vélez, S. \& Castilla, P. (2018). Revisión del Marco de Sendai, una reflexión crítica sobre el desarrollo y la gestión del riesgo de desastres. Monografía. Manizales: Universidad Católica de Manizales. Recuperado de: http://repositorio.ucm.edu.co:8o8o/jspui/bitstream/handle/10839/2255/ Santiago\%2oVelez\%2oToro.pdf?sequence=1\&isAllowed=y [visitado o4 de agosto de 2019] 